\section{Mutations and oncogenes - cause or effect}

SIR - In a field as complex as carcinogenesis, it is difficult to distinguish between cause, correlation and consequence. No thoughtful biologist needs to be reminded of the many cancer theories propounded in this century. The current version is represented by the flood of papers on oncogenes and karyotypic abnormalities as the underlying cause of cancer, with amplification in the popular press.

The problem in unqualified acceptance of the claims can be illustrated by an examination of some details of specific and nonspecific chromosome changes in cancer. The most thoroughly investigated of the specific or nonrandom changes is the Philadelphia chromosome $\left(\mathrm{Ph}^{1}\right)$ associated with over $90 \%$ of human cases of chronic myelogenous leukaemia $(\mathrm{CML})^{1-3}$. However, there are patients with typical CML whose leukaemic marrow cells are $\mathrm{Ph}^{1}$ negative at first, but become $\mathrm{Ph}^{1}$ positive later ${ }^{4}$. In one case, $\mathrm{Ph}^{1}$ negative and $\mathrm{Ph}^{1}$ positive cells were observed in the same clone of leukaemic cells ${ }^{5}$. The evidence suggests that blood cells remain karyotypically normal but unstable for some time after the initial malignant transformation but provide a milieu favourable for the establishment of the $\mathrm{Ph}^{1}$ anomaly ${ }^{5}$.

Similar evolution of nonrandom chromosome alteration occurs in sarcomas induced in rats by infection with the Schmidt-Ruppin strain of Rous sarcoma virus. More than $80 \%$ of the tumours have a normal diploid stemline ${ }^{6}$. In some cases, all the cells are normal diploid while in others there are a few near-diploid sidelines or pseudodiploids. In older tumours, however, heteroploidy is common and usually involves the specific, sequential addition of three particular chromosomes. Without a careful impression that the chromosonal aberrations are a prerequisite for tumour formation since the predominance of normal diploid cells in early tumours is sometimes not noted when the nonrandom changes are discussed ${ }^{7}$.

Many of the reports of nonrandom chromosome changes or activated oncogenes in tumours are based on studies of cultured lines of the tumour cells. The caution that must be used in interpreting them is illustrated by a study of eight human gliomas ${ }^{8}$, each of which had a unique and broad distribution of karyotypes ranging from hypodiploid to as much as hypertetraploid when first placed in culture. Reference sets of frequent karyotypes were established for each of the tumours, only 7 to $25 \%$ of the clonal karyotypes were represented in the reference sets. Thus in no case were the cells which were maintained in culture representative of the original tumour, and any conclusions drawn from their analysis could not be applied to the bulk of the tumour cells. All of the examples given demonstrate that progression within the tumour and selection in culture obscure the relationship between cause and effect.

Chromosome studies have an advantage over molecular analysis of oncogene analysis because karyotypes can be obtained of individual cells, and a population distribution determined, whereas the biological and chemical methods used to identify oncogenes represent an average of the whole of the population. Furthermore, karyotype analysis is technically simple and has been pursued even in clinical laboratories for many years, so it has provided a depth of information, particularly in human cancer, not yet available in oncogene studies.

There are, however, hints that the questions raised about the role of chromosome changes in tumour development have their parallels in studies of oncogenes. Most of the positive reports of oncogene activation derive from transfection studies with DNA from cell lines9. (Chemical identification of activated oncogenes in some 50 reported cases of human cancer has been negative ${ }^{9}$ ). The most prominent cellular oncogenes are related to acute viral transforming genes which are known to give a selective advantage for cell growth in culture. The possibility must therefore be considered that cell structure selects for a minority mutant cell in the original tumour population. The activated oncogene would then be no more representative of the tumour cells than was the clonal karyotype in the glioma representative of the tumour karyotype.

It has recently been shown that a human osteosarcoma cell line which was negative on transfection to NIH3T3 cells could become positive after treatment with $N$-methyl. $V^{i}$-nitro- $N$-nitrosoguanidine (MNNG), a mutagen and carcinogen ${ }^{10}$. The chemically-transformed cells were more transformed in appearance than the original cancer cells and produced more tumours in nude mice. Obviously the "oncogene activation" was an epiphenomenon unrelated to the original tumour. Although this activation may have played a role in the secondary transformation of the tumour cells in culture by MNNG, even this is uncertain, since a similar enhancement of the transformed phenotype in the same cancer cells by another carcinogen was not accompanied by detectable onocogene activation. Many more inconsistencies in the ever burgeoning oncogene literature could be cited to raise suspicion about their purported role in carcinogenesis (see ref. 9). At the most, their role in tumorigenesis appears to be auxiliary ${ }^{11}$, at the least inconsequential.

As confirmed recently in Nature, the initial effects of carcinogenic treatment affect the entire population of exposed cells $^{12}$ and therefore cannot be genetic. The rarer, random occurences which characterize the secondary stages of transformation ${ }^{12}$ can be explained as well by differentiative ${ }^{13}$ as by mutational events. Indeed, the great mass of (largely dormant) evidence which indicates that cancer is the far end of a spectrum of development of cell and tissues ${ }^{14,15}$ seems to be only selectively disinterred to rationalize gaps in mutational theories of cancer. The lack of an adequate mechanistic explanation for developmental processes and their derangement is no justification for ignoring them.

Department of Molecular Biology, HARRY RUBIN

University of California,

Berkeley, California 94720, USA

1. Nowell, P. C. \& Hungerford, D. A. Science 132, 1497 (1960).

2. Rawley, J. B. Nature 243, 290-293 (1973)

3. Wolman, S. Cancer Metastasis Rev. 2, 257-293 (1983).

4. Lisker, R. L., Casas, L., Mutchnick, O., Perez-Chavez, F. \& Labardini, J. Blood 56, 812-823 (1983).

5. Fialkow, P. J., Martin, P. J., Najfield, V., Penfold, G. K. Jacobsen, R. J. \& Hansen, A. Blood 58, 158-163 (1981). Mitelman, F. Hereditas 69, 155-186 (1971)

7. Mitelman, F. Mark, J., Levan, G. \& Levan, A. Science Mitelman, F., Mark, J.,
176, 1340-1341 (1972).

8. Shapiro, J. R. Yung, W-K. A. \& Shapiro, W. R. Cancer Res. 41, 2349-2359 (1981).

9. Duesberg, P. Nature 304, 219-225 (1983)

10. Cooper, C. S. et al. Cancer Res. 44. 1-10 (1984).

11. Land, H., Parada, L. F. \& Weinberg, R. A. Nature 304, 596-602 (1983).

12. Kennedy, A. R., Cairns, J. \& Little, J. H. Nature 307, 85-86 (1984).

13. Nakano, S. \& Tso, P.O. Proc. natn. Acad. Sci. USA 78, 4995-4999 (1981).

14. Smithers, D. W. Lancet i, $493-499$ (1962).

15. Foulds, L. Neoplastic Development Vols 1 \& II (Academic, New York).

\section{Oligodendroglia in multiple sclerosis}

SIR - The report by Ludwin ${ }^{1}$ that the oligodendrocyte proliferates around an injury to mouse brain is of great relevance to previous studies on the neuroglia in mutliple sclerosis (MS). Some twenty years ago, we demonstrated proliferation (or at least increased numbers) of oligodendroglia at the edge of active MS plaques ${ }^{2,3}$. This observation has recently been confirmed by electron microscopy ${ }^{4}$.

Although oligodendroglia are essentially absent from the centre of MS plaques ${ }^{2.3 .5}$, their proliferation at the edge of the lesion suggests either a response to injury or their involvement in repair of the damaged myelin. Ludwin's dynamic isotope study clarifies the controversial issue of the oligodendrocyte's potential to undergo hyperplasia and possible role in repair.

C.W.M. ADAMS

Department of Pathology,

Guy's Hospital Medical School, London SE1 9RT, UK

Ludwin, S.K. Nature 308, 274 (1984)

. Ibrahim, M.Z.M. \& Adams, C.W.M. J. Neurol. Neurosurg. Psychiat. 26, I0I (1963).

3. Ibrahim, M.Z.M. \& Adams, C.W.M. J. Pathol. Bact. 90 239 (1965).

4. Raine, C. in Multiple Sclerosis (eds Hallpike, J.F., Adams, C.W.M. \& Tourtelloti, W.W.) 413-460 (Chapman and Hall, I ondon, 1983).

5. L.umsden, C.E. Br. Med. J. i, 1035 (1951). 